\title{
Buckling modes of elastic thin films on elastic substrates
}

Haixia Mei and Rui Huang*

Department of Aerospace Engineering and Engineering Mechanics, University of Texas, Austin, $T X 78712$

Jun Young Chung and Christopher M. Stafford

Polymers Division, National Institute of Standards and Technology, Gaithersburg, MD 20899

Hong-Hui Yu

Department of Mechanical Engineering, The City College of the City University of New York, New York, NY 10021

\begin{abstract}
Two buckling modes have been observed in thin films: buckle-delamination and wrinkling. This Letter identifies the conditions for selecting the favored buckling modes for elastic films on elastic substrates. Transition from one buckling mode to another is predicted as the stiffness ratio between the substrate and the film or the interfacial defect size varies. The theoretical results are demonstrated experimentally by observing the coexistence of both buckling modes and mode transition in one film-substrate system.
\end{abstract}

\footnotetext{
*Electronic mail: ruihuang@mail.utexas.edu
} 
Thin film materials are used in a wide range of applications including microelectronics, coatings, and medical devices. A frequent failure mechanism in these materials is buckling of the thin films, resulting in interfacial delamination and fracture [1,2]. Recently, understanding of buckle patterns has also led to applications in metrology [3-5], stretchable interconnects [6,7], and optical gratings [8]. Previous studies on thin film buckling have focused on one of the two buckling modes, buckle-delamination or wrinkling, as illustrated in Fig. 1. The former considers buckling of the film when it is partly delaminated from the substrate $[9,10]$, while the latter assumes no delamination as the substrate deforms coherently with the film [11-13]. The characteristics of both buckling modes are often observable, with localized patterns for buckledelamination (e.g., telephone cord blisters) and homogeneous patterns for wrinkling. One of the unanswered questions is: what determines the selection of either buckling mode for a given material system? Intuitively, buckle-delamination typically occurs with relatively stiff substrates and wrinkling occurs only when the substrate is very soft. However, a quantitative criterion is lacking. In this Letter, we develop a theoretical understanding of the buckling mode selection for elastic thin films on elastic substrates. This is demonstrated experimentally by observing both the buckling modes and a mode transition in a single film-substrate system with varying stress and interfacial defects.

As a stress-driven instability, the study of buckling can be traced back to the classical Euler buckling of an elastic column. The fundamental buckling mode minimizes the elastic strain energy and depends on the type of constraints. For a thin elastic film bonded to a thick elastic substrate, the buckling instability is constrained by the substrate. Without delamination, buckling of the film (i.e., wrinkling) requires coherent deformation of the substrate, which is possible only 
when the substrate is relatively compliant. Based on an energetic analysis [11-13], the critical stress for wrinkling is:

$$
\sigma_{w}=\frac{\bar{E}_{f}}{4}\left(\frac{3 \bar{E}_{s}}{\bar{E}_{f}}\right)^{2 / 3},
$$

where $\bar{E}_{f}$ and $\bar{E}_{s}$ are the plane-strain moduli of the film and substrate, respectively. When the compressive stress in the film, $\sigma>\sigma_{w}$, the film buckles spontaneously, forming wrinkles throughout the film surface. A particular wrinkle wavelength is established to minimize the total elastic energy in the film and the substrate [11-13]. The effects of substrate and film thicknesses on the wrinkling stress have also been studied previously [14,15].

For stiff substrates, buckling deformation of the film is highly constrained, leading to high critical stresses for wrinkling. However, the substrate constraint may be locally mitigated by interfacial defects that lead to partial delamination of the film. In this case, the delaminated portion of the film buckles, which in turn drives growth of delamination through interfacial fracture $[9,10]$. The co-development of buckling and delamination leads to abundant blister patterns such as telephone-cord blisters. Compared to wrinkling, the buckle-delamination patterns are typically localized and sensitive to interfacial defects [16,17]. Early studies of buckle-delamination often assumed a fixed-end condition at the edge of delamination, which essentially neglected the effect of elastic deformation in the substrate. Under such a condition, the critical stress for the onset of buckling is identical to that for a free-standing sheet [9], i.e.,

$$
\sigma_{B 0}=\frac{\pi^{2}}{12}\left(\frac{h}{b}\right)^{2} \bar{E}_{f},
$$


where $h$ is the film thickness and $b$ is the half width of the delamination. The buckling stress $\sigma_{B 0}$ is independent of the substrate properties but depends on the relative size of the interfacial delamination, $b / h$.

Recent studies [18-20] have shown that the buckling stress can be significantly lower than that predicted by Eq. (2) when the elastic deformation of the substrate is considered, especially for compliant substrates. Yu and Hutchinson [20] derived an implicit expression

$$
\sqrt{\frac{\sigma_{B 0}}{\sigma_{B}}} \tan \left(\pi \sqrt{\frac{\sigma_{B}}{\sigma_{B 0}}}\right)=\frac{\pi h}{12 b}\left(\frac{a_{12}^{2}}{b / h+a_{11}}-a_{22}\right),
$$

where $a_{11}, a_{22}$, and $a_{12}$ are determined numerically, either by direct finite element calculations or by solving an integral equation, as dimensionless spring constants at the edge of the buckled film, which depend on the ratio $b / h$ and two Dundurs' parameters. The first Dundurs' parameter, $\alpha$, is related to the stiffness ratio as $\bar{E}_{s} / \bar{E}_{f}=(1-\alpha) /(1+\alpha)$. The second Dundurs' parameter, $\beta$, typically plays a less important role than $\alpha$ [20]. In this study, we concentrate on the effect of $\alpha$ and take $\beta=0$ by setting Poisson's ratios $v_{f}=v_{s}=0.5$ in the numerical calculations.

A comparison between the critical stresses for wrinkling and buckle-delamination is presented in Fig. 2. The stresses are normalized by the plane-strain modulus of the film and plotted versus the substrate-film stiffness ratio. Using the log-log scales, the critical wrinkling stress in Eq. (1) is a straight line with a slope 2/3. The critical stress for buckling, $\sigma_{B}$, obtained from Eq. (3), decreases as the relative delamination size $b / h$ increases. For a constant delamination size, the buckling stress increases as the stiffness ratio increases, but at a slower rate compared to the increase of the wrinkling stress. The intersection of the two critical stresses defines a critical stiffness ratio, $R_{c}$. When the relative substrate stiffness is greater (i.e., $\bar{E}_{s} / \bar{E}_{f}>R_{c}$ ), the buckling stress is lower than the wrinkling stress, dictating that buckle- 
delamination occurs first as the compressive stress develops in the film. On the other hand, for more compliant substrates $\left(\bar{E}_{s} / \bar{E}_{f}<R_{c}\right)$, the wrinkling stress is lower and the film wrinkles. Therefore, a transition in the buckling mode is predicted quantitatively as the stiffness ratio between the substrate and the film varies. The critical value $R_{c}$ as a function of the delamination size is plotted in Fig. 3. This plot represents a buckling mode selection map: if the stiffness ratio and the interfacial defect size render a point below the $R_{c}$ curve, wrinkling is energetically favored; otherwise, buckle-delamination is favored. It should be noted that the present discussion is limited to the onset of the initial buckling mode only.

We note that, for each delamination size, the buckling stress determined from Eq. (3) has two limits: for high substrate/film stiffness ratios, the buckling stress approaches $\sigma_{B 0}$ as given by Eq. (2); for very low stiffness ratios, the buckling stress approaches $\sigma_{B 0} / 4$. These limiting stresses are plotted as horizontal dashed lines in Fig. 2. While the upper limit corresponds to the buckling of the delaminated film with fixed ends as an approximation for a rigid substrate, the lower limit is identical to that for buckling of a free-standing film of length $2 b$ simply supported at both ends. Therefore, as the relative substrate stiffness decreases, the effective constraint at the edges of delamination relaxes from essentially fixed ends (constraint on both displacement and rotation) to simple supports (no constraint on rotation). This is reasonable if one considers buckling of the delaminated region only. However, as discussed above, when $\bar{E}_{s} / \bar{E}_{f}<R_{c}$, buckling is no longer limited to the delaminated region. Consequently, Eq. (3) should be valid only for the regime where $\bar{E}_{s} / \bar{E}_{f}>R_{c}$. Another limit for Eq. (3) is set by the use of the plate equations for the delaminated part, which roughly requires $b / h>3$. 
To validate the buckling mode selection criterion described above, we designed an experiment that enabled observation of both the buckling modes as well as a mode transition. Here, a polystyrene (PS) film ( $h=120 \mathrm{~nm}$ ) was bonded to a $1 \mathrm{~mm}$ thick polydimethylsiloxane (PDMS) substrate. The materials' elastic moduli are estimated as $\bar{E}_{f} \sim 4 \mathrm{GPa}$ and $\bar{E}_{s} \sim 2 \mathrm{MPa}$, with a ratio $\bar{E}_{s} / \bar{E}_{f}=0.0005$ [3]. From Eq. (1), the critical stress for wrinkling is $\sigma_{w} \sim 13 \mathrm{MPa}$. By annealing the specimen at $120^{\circ} \mathrm{C}$, which is above the glass transition temperature of PS ( $T_{g} \sim$ $105^{\circ} \mathrm{C}$ ), and then slowly cooling, a compressive stress develops in the PS film due to differential thermal expansion. Assuming an initially small defect at the interface (say, $b / h=5$ ), as illustrated by the vertical line in Fig. 2, the compressive stress in the PS film first reaches the wrinkling stress, at which point wrinkling occurs spontaneously over the film surface. As the temperature is further decreased, the stress eventually reaches the critical buckling stress for the initial defect size, and buckle-delamination occurs alongside with the existing wrinkles. Figure 4 shows a set of optical images of the PS film on the PDMS substrate, which demonstrates the occurrence of wrinkling followed by buckle-delamination during the first cooling cycle (top row). The inset Fourier spectrum shows a well-defined wrinkle wavelength at $60{ }^{\circ} \mathrm{C}$. When the material system was heated back to $120{ }^{\circ} \mathrm{C}$ from the room temperature, the compressive stress in the film vanished and the elastic film recovered with no observable buckling or wrinkling. Next, the system was subjected to a second cooling (Fig. 4, second row). Compared to the first cooling, the interfacial defect size has increased due to the growth of buckle-delamination in the first cycle. An estimate of the delamination width (Fig. 4, top row, $35^{\circ} \mathrm{C}$ ) gives $b / h \sim 20$ for the second cycle, for which the buckling stress is lower than the wrinkling stress with $\bar{E}_{s} / \bar{E}_{f}=0.0005$ as shown in Fig. 2. Consequently, buckle-delamination occurred first, at locations similar to those in the first cycle. Further cooling led to growth of buckle-delamination and eventually wrinkling 
of the bonded region as well. Different buckling and wrinkling patterns are observed for the first and second cycles, which can be attributed to the different evolution paths for mode transition and interactions.

In summary, by comparing the critical stresses for wrinkling and buckle-delamination, we develop a quantitative criterion for the selection of initial buckling modes in elastic thin films. A buckling mode selection map is constructed with respect to the stiffness ratio and the interfacial defect size. The theoretical result is demonstrated by an experiment that observed the coexistence of both buckling modes and a mode transition.

\section{Acknowledgments}

HM and RH are grateful for the financial support by National Science Foundation under Grant No. 0547409. Portion of this work was conducted at the National Institute of Standards and Technology Combinatorial Methods Center. This manuscript is an official contribution of the National Institute of Standards and Technology, not subject to copyright in the United States. 


\section{References}

1. J.W. Hutchinson, M.D. Thouless, E.G. Liniger, Acta Metall. Mater. 40, 295 (1992).

2. H. Yin, R. Huang, K.D. Hobart, J. Liang, Z. Suo, S.R. Shieh, T.S. Duffy, F.J. Kub, J.C. Sturm, J. Appl. Phys. 94, 6875 (2003).

3. C.M. Stafford, C. Harrison, K.L. Beers, A. Karim, E.J. Amis, M.R. VanLandingham, H.-C. Kim, W. Volksen, R.D. Miller, E.E. Simonyi, Nat. Mater. 3, 545 (2004).

4. E.A Wilder, S. Guo, S. Lin-Gibson, M.J. Fasolka, C.M. Stafford, Macromolecules 39, 4138 (2006).

5. C.M. Stafford, B.D. Vogt, C. Harrison, D. Julthongpiput, R. Huang, Macromolecules 39, 5095 (2006).

6. M. Watanabe, H. Shirai, T. Hirai, J. Appl. Phys. 92, 4631 (2002).

7. S.P. Lacour, S. Wagner, Z.Y. Huang, Z. Suo, Appl. Phys. Lett. 82, 2404 (2003).

8. C. Harrison, C.M. Stafford, W. Zhang, A. Karim, Appl. Phys. Lett. 85, 4016 (2004).

9. J.W. Hutchinson and Z. Suo, Adv. Appl. Mech. 29, 63 (1992).

10. M. Ortiz and G. Gioia, Adv. Appl. Mech. 33, 119 (1997).

11. T.W. Shield, K.S. Kim, R.T. Shield, J. Appl. Mech. 61, 231 (1994).

12. J. Groenewold, Physica A298, 32 (2001).

13. Z.Y. Huang, W. Hong, Z. Suo, J. Mech. Phys. Solids 53, 2101 (2005).

14. R. Huang, J. Mech. Phys. Solids 53, 63 (2005).

15. R. Huang, C.M. Stafford, B.D. Vogt, J. Aerospace Engineering 20, 38 (2007).

16. M.-W. Moon, J.-W. Chung, K.-R. Lee, K.H. Oh, R. Wang, A.G. Evans, Acta Mater. 50, 1219 (2002).

17. C. Coupeau, Thin Solid Films 406, 190 (2002). 
18. B. Cotterell and Z. Chen, Int. J. Fracture 104, 169 (2000).

19. G. Parry, J. Colin, C. Coupeau, F. Foucher, A. Cimetiere, J. Grilhe, Acta Mater. 53, 441 (2005).

20. H.H. Yu and J.W. Hutchinson, Int. J. Fracture 113, 39 (2002). 


\section{List of Figures}

Fig. 1: (Color online) Schematic of two buckling modes in thin films. The critical stresses, $\sigma_{w}$ and $\sigma_{B}$, are given by Eqs. (1) and (3), respectively.

Fig. 2: (Color online) Comparison between the critical stresses for wrinkling and buckledelamination. The open symbols are numerical results from Eq. (3) for various $b / h$ ratios. The dashed lines indicate the limiting stresses for buckle-delamination. The vertical line represents the PS/PDMS system with $\bar{E}_{s} / \bar{E}_{f}=0.0005$.

Fig. 3: The critical stiffness ratio as a function of the relative delamination size. Below the line, wrinkling is favored; above the line, buckle-delamination is favored. The dashed portion shows the qualitative trend beyond the limit of the plate theory used in deriving Eq. (3).

Fig. 4: Optical micrographs of a $120 \mathrm{~nm}$ PS thin film on a PDMS substrate cooled from $120^{\circ} \mathrm{C}$ to room temperature, where wrinkling is the favored buckling mode during the $1^{\text {st }}$ cooling cycle (top row) and buckle-delamination is favored in the $2^{\text {nd }}$ cycle. The Fourier spectrum at $60{ }^{\circ} \mathrm{C}$ (inset) in the first cycle shows a well-defined wrinkle wavelength. 


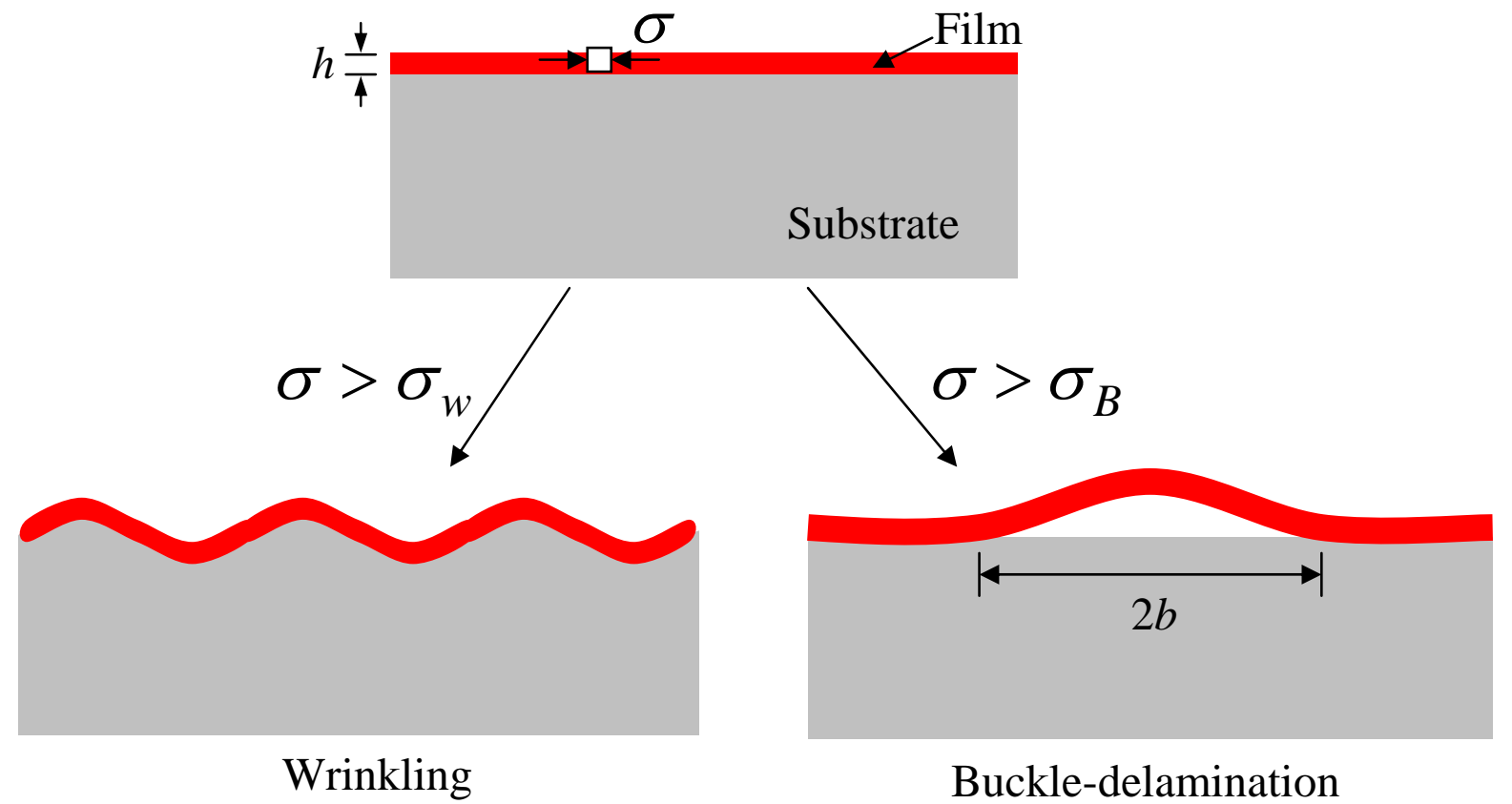

Fig. 1: (Color online) Schematic of two buckling modes in thin films. The critical stresses, $\sigma_{w}$ and $\sigma_{B}$, are given by Eqs. (1) and (3), respectively. 


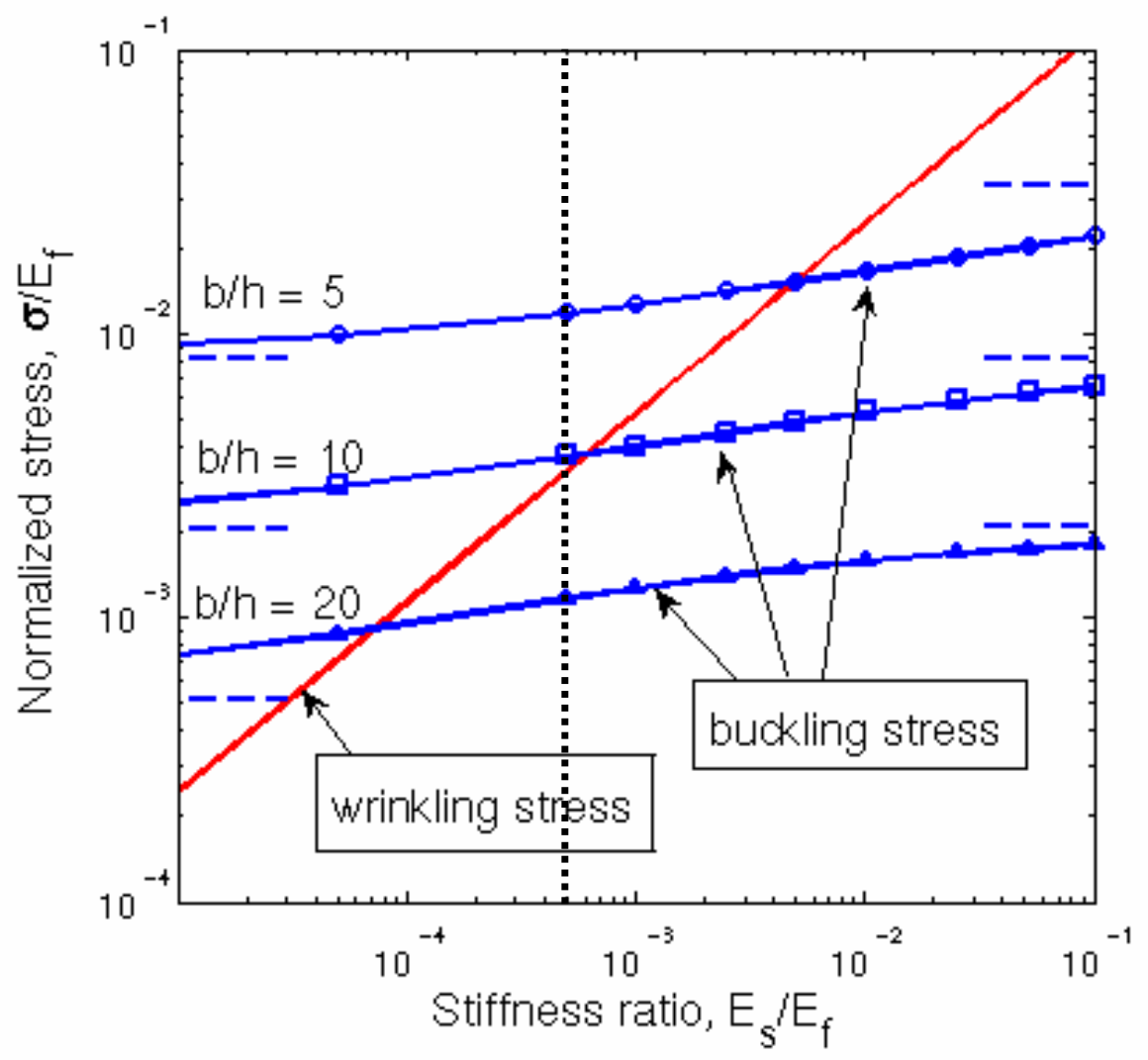

Fig. 2: (Color online) Comparison between the critical stresses for wrinkling and buckledelamination. The open symbols are numerical results from Eq. (3) for various $b / h$ ratios. The dashed lines indicate the limiting stresses for buckle-delamination. The vertical line represents the PS/PDMS system with $\bar{E}_{s} / \bar{E}_{f}=0.0005$. 


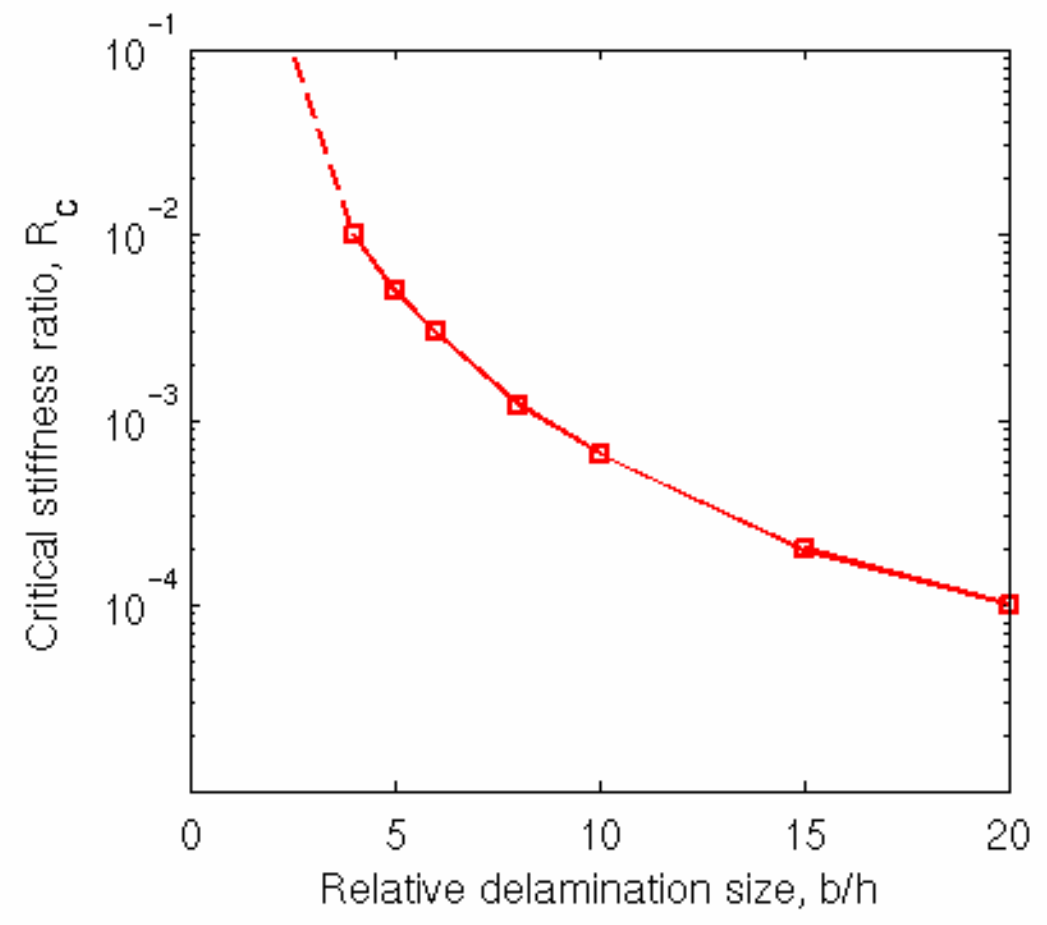

Fig. 3: The critical stiffness ratio as a function of the relative delamination size. Below the line, wrinkling is favored; above the line, buckle-delamination is favored. The dashed portion shows the qualitative trend beyond the limit of the plate theory used in deriving Eq. (3). 


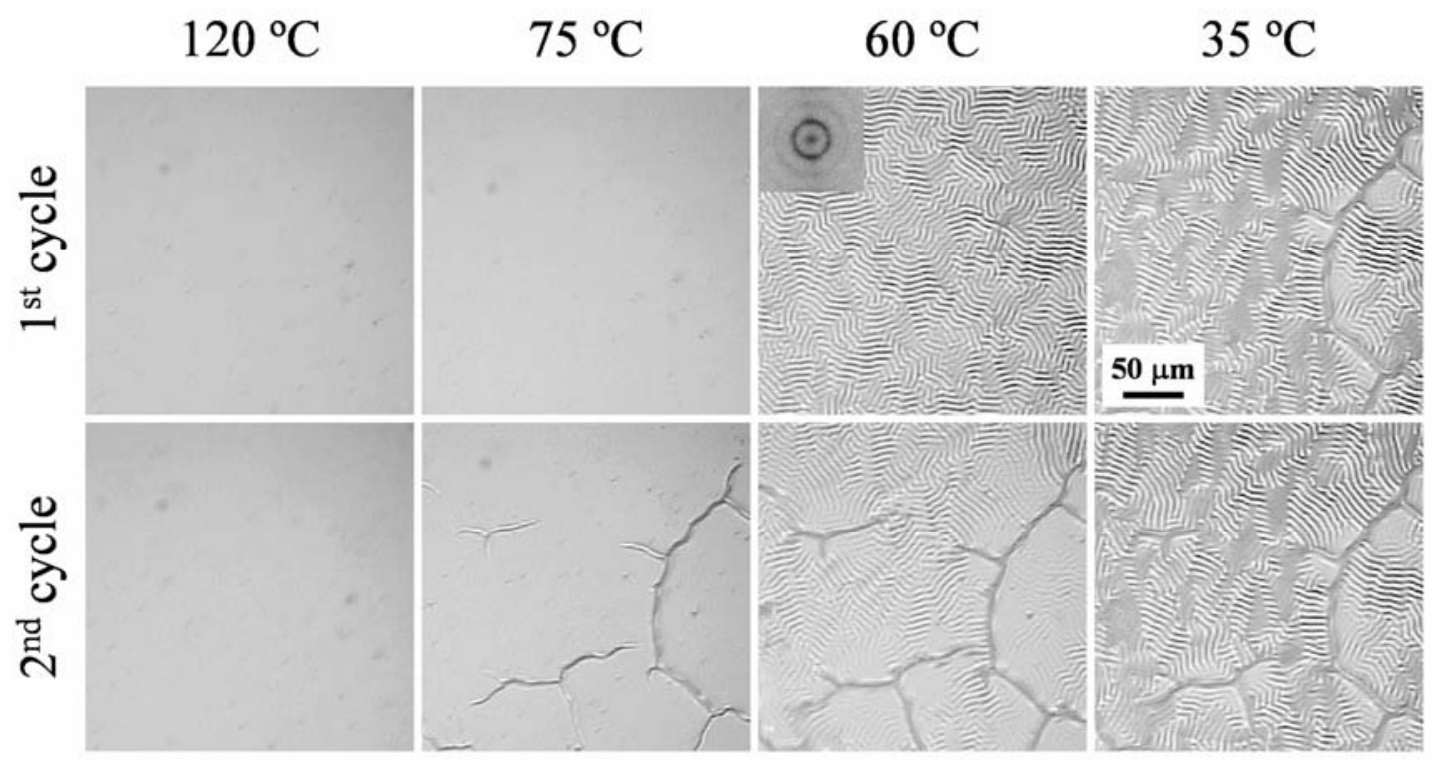

Fig. 4: Optical micrographs of a $120 \mathrm{~nm}$ PS thin film on a PDMS substrate cooled from $120{ }^{\circ} \mathrm{C}$ to room temperature, where wrinkling is the favored buckling mode during the $1^{\text {st }}$ cooling cycle (top row) and buckle-delamination is favored in the $2^{\text {nd }}$ cycle. The Fourier spectrum at $60{ }^{\circ} \mathrm{C}$ (inset) in the first cycle shows a well-defined wrinkle wavelength. 